\section{Pressão arterial elevada em adolescentes e fatores associados: um estudo de base escolar em Uruguaiana, Rio Grande do Sul, 2011}

\section{High arterial blood pressure in adolescents and associated factors: a school-based study in Uruguaiana, Rio Grande do Sul, 2011}

Mauren Lúcia de Araújo Bergmann 1 Susane Graup 2

Gabriel Gustavo Bergmann 3
1-3 Universidade Federal do Pampa. BR 472 - Km 592. Caixa Postal 118. Uruguaiana, RS, Brasil. CEP: 97.508-000.

E-mail: gabrielbergmann@unipampa.edu.br

\begin{abstract}
Objectives: to identify the prevalence of high arterial blood pressure $(A B P)$ and associated factors in adolescents from Uruguaiana (RS), 2011.

Methods: a probabilistic study was carried out with adolescents aged between 10 and 17 years enrolled in public schools. The ABP was measured by auscultation and classified as normal or high (ABP above the 90th percentile). Socio-demographic, psychosocial and behavioral factors, nutritional status and physical condition were assessed. A logistic regression analysis was carried out using a hierarchical theoretical model.

Results: the study covered 1455 adolescents (741 female). The prevalence of high systolic and diastolic $A B P$ was $16.4 \%$ (C195\%: 14.4-18.4) and $18.5 \%$ (CI95\%: 16.4-20.6), respectively. The factors that remained associated with high systolic and diastolic $A B P$ in the final model were: perceiving oneself to be less fit than one's peers (systolic OR: 2.27; CI95\%: 1.22-4.23; diastolic OR: 2.99; CI95\%: 1.34-6.70) and overweight and obesity (systolic OR: 9.29; C195\%: 4.89-17.69; diastolic OR: 4.70; CI95\%: 2.61-8.45). Female adolescents (OR: 1.39; CI95\%: 1.02-1.90) and those of a higher socio-economic status (OR: 2.39; CI95\%: 1.31-4.36) were more likely to present with high systolic $A B P$.

Conclusions: the prevalence of high ABP found in adolescents was high. To prevent this, programs to combat excess weight should be designed, taking into consideration socio-demographic characteristics such as sex and socio-economic status.
\end{abstract}

Key words Students, Hypertension, Risk factors, Motor activity, Obesity, Cross-sectional studies
Resumo

Objetivos: identificar a prevalência de pressão arterial (PA) elevada e seus fatores associados em adolescentes de Uruguaiana (RS), 2011.

Métodos: amostra probabilística composta por adolescentes de 10 a 17 anos de escolas públicas. A PA foi aferida pelo método auscultatório e classificada em normal ou elevada (PA acima do percentil 90). Fatores sociodemográficos, psicossociais, comportamentais, estado nutricional e aptidão física foram analisados. Foi realizada uma análise de regressão logística considerando um modelo teórico hierárquico.

Resultados: participaram do estudo 1455 adolescentes (741 do sexo feminino). A prevalência de $P A$ sistólica e PA diastólica elevadas foram de 16,4\% (IC95\%: 14,4-18,4) e de 18,5\% (IC95\%: 16,4-20,6), respectivamente. Permaneceram associados à $P A$ sistólica e diastólica elevadas no modelo final: se perceber menos em forma que seus pares (sistólica OR: 2,27; IC95\%: 1,22-4,23; diastólica OR: 2,99; IC95\%: 1,34-6,70) e o sobrepeso e obesidade (sistólica OR: 9,29; IC95\%: 4,89-17,69; diastólica OR: 4,70; IC95\%: 2,61-8,45). Ainda, adolescentes do sexo feminino (OR: 1,39; IC95\%: 1,02-1,90) e de maior nivel socioeconômico (OR: 2,39; IC95\%: 1,314,36) têm mais chance de apresentarem PA sistólica elevada.

Conclusões: a prevalência de PA elevada encontrada entre os adolescentes é alta. Para sua prevenção, programas de combate ao excesso de peso considerando características sociodemográficas como o sexo e nível socioeconômico devem ser elaboradas.

Palavras-chave Estudantes, Hipertensão, Fatores de risco, Atividade motora, Obesidade, Estudos transversais 


\section{Introdução}

As doenças cardiovasculares são a principal causa de morte na população adulta e a hipertensão arterial, enfermidade que atinge cerca de $20 \%$ dessa população mundial (maior de 18 anos), é um dos fatores de risco mais importantes. ${ }^{1} \mathrm{O}$ Brasil parece seguir a tendência mundial, tendo $22 \%$ dos indivíduos acima de 18 anos com níveis elevados de pressão arterial (PA). ${ }^{1}$

Embora a hipertensão arterial geralmente se manifeste na fase adulta da vida, ${ }^{2}$ o problema parece ter sua origem a infância e adolescência. ${ }^{3}$ Os elevados níveis pressóricos ocasionam o aumento da espessura da íntima média da artéria carótida ${ }^{4} \mathrm{e}$ diminuem a elasticidade arterial, 5 estando igualmente associada à hipertrofia do ventrículo esquerdo. ${ }^{6}$ Com base nessas evidências, supõe-se que a hipertensão no início da vida pode ser um preditor de hipertensão no adulto.

Considerando a elevada prevalência de hipertensão na população nos dias atuais, pode-se supor que em muitos casos indivíduos jovens deixaram de ser diagnosticados nas últimas décadas e, dessa forma, o monitoramento regular da PA de crianças e adolescentes tem sido sugerido.7 Este acompanhamento pode contribuir para que medidas preventivas sejam instituídas já nos primeiros anos de vida. Além disto, a identificação dos fatores associados à PA elevada é fundamental para a concepção destas medidas.

Alguns estudos8-11 brasileiros com amostras representativas sobre a associação conjunta de diferentes fatores com a PA em adolescentes foram desenvolvidos. Contudo, devido à diversidade de fatores analisados e à disparidade nos resultados encontrados, com exceção dos indicadores antropométri-cos de sobrepeso e obesidade, ${ }^{8-11}$ ainda não é possível estabelecer com precisão, os fatores de risco para PA elevada nesta população. ${ }^{3}$ Dentre os fatores estudados, apenas os indicadores antropométricos de sobrepeso e obesidade se associaram de forma direta à PA elevada em todos os estudos. ${ }^{8-11}$ Diante disto, o objetivo deste estudo foi identificar a prevalência PA elevada e seus fatores associados em adolescentes.

\section{Métodos}

Este estudo transversal, de base escolar, utilizou dados de uma pesquisa conduzida em escolares do turno diurno da rede de ensino pública da cidade de Uruguaiana no Rio Grande do Sul, com o objetivo de estimar a prevalência e os fatores associados à inatividade física em escolares de 10 a 17 anos, de abril a novembro do ano de 2011.

O cálculo do tamanho da amostra foi realizado considerando os seguintes dados: número de alunos matriculados regularmente no ano de 2011 na faixa etária do estudo $(\mathrm{n}=15.210)$, prevalência de $50 \%$ (considerando um estudo com múltiplos desfechos), Intervalo de Confiança de 95\%, erro aceitável de 3\% e percentual de perdas e recusas de $20 \%$, obtendo-se um $\mathrm{n}=1196$.

O processo de seleção dos adolescentes foi probabilístico em múltiplos estágios. Com o intuito de garantir a representatividade e proporcionalidade de alunos por regiões, inicialmente o município foi dividido em cinco áreas geográficas, quatro na zona urbana (quadrantes) e uma representativa da zona rural. Nove escolas da zona urbana e uma da zona rural foram sorteadas. Todas as escolas das cinco áreas tiveram a mesma chance de ser sorteadas.

Todos os escolares com idade entre 10 a 17 anos matriculados nas 10 escolas selecionadas foram convidados a participar. Foram excluídos os estudantes que apresentassem algum tipo de deficiência física ou cognitiva que comprometesse a utilização de suas informações e aqueles que fizessem uso de algum medicamento que influenciasse nos níveis de PA.

A coleta dos dados foi feita utilizando questionário estruturado autoaplicável para a obtenção de informações sociodemográficas, psicossociais e comportamentais. No mesmo instrumento foram registrados pelos avaliadores os valores de PA sistólica (PAS), de PA diastólica (PAD), do estado nutricional e da aptidão física.

As medidas de PAS e PAD foram obtidas por uma equipe previamente treinada para a aferição da PA pelo método auscultatório. Todos os instrumentos utilizados (estetoscópios, manguitos e esfigmomanômetros) eram certificados pelo Instituto Nacional de Metrologia. Foram utilizados manguitos e esfigmomanômetros nas dimensões adequadas ao tamanho do braço e os adolescentes foram instruídos a não realizarem atividade física (AF) nos 30 minutos anteriores à aferição. A aferição da PA foi realizada em triplicata obedecendo a um intervalo de aproximadamente dois minutos entre a obtenção das medidas. Os adolescentes deviam estar em repouso prévio de no mínimo dez minutos. As mensurações foram realizadas no braço direito, em posição sentada e em situação de relaxamento. A PAS foi determinada no momento da ausculta do primeiro som (fase I do som de Korotkof) e a PAD obtida no momento do desaparecimento do mesmo (fase $\mathrm{V}$ do som de Korotkof). Para este estudo, foram conside- 
rados os valores de PAS e PAD obtidos na última medida.

A categorização da PA foi realizada de acordo com The Fourth Report on Diagnosis, Evaluation, and Treatment of High Blood Pressure in Children and Adolescents. 12 Os adolescentes foram classificados em normalidade (PA inferior ao percentil 90), pré-hipertensão (PA percentil 90 a 95) e hipertensão (PA acima do percentil 95). Para a análise, os valores PAS e PAD classificados como pré-hipertenso e hipertenso foram agrupados em uma categoria classificada como PAS e PAD elevadas.

As variáveis independentes foram organizadas em quatro blocos hierárquicos:

Sociodemográficas: sexo (masculino ou feminino), idade em anos completos divididos em quatro grupos etários (10-12 anos; 13-15 anos; 16-17 anos), nível socioeconômico (classes A, B, C, D e E, conforme a Classificação Econômica Brasil13), zona de residência (urbana ou rural) e tipo de residência (casa ou apartamento).

Psicossociais: gostar das aulas de educação física (EF) (sim ou não); gostar de praticar AF (sim ou não); percepção sobre a forma corporal (muito mais em forma que os outros; mais em forma que os outros; igualmente em forma; menos em forma que os outros; ou completamente fora de forma); percepção sobre o nível de AF (muito menos ativo que os outros; menos ativo que os outros; igualmente ativo aos outros; mais ativo que os outros; ou muito mais ativo que os outros)

Comportamentais: deslocamento até a escola (ativo: a pé, de bicicleta, ou outra forma de deslocamento que dependesse de esforço físico; ou passivo: uso de algum tipo de veículo automotor); prática de EF escolar (sim ou não); AF com orientação (por um responsável) além da EF escolar ( $\leq 1 \mathrm{vez} / \mathrm{semana}$; ou $\geq 2$ vezes/semana); tempo de tela (tempo médio diário utilizando televisão, jogos eletrônicos e computador): $\leq 3$ horas ou $>3$ horas; tabagismo (nunca fumei; fumei, mas não fumo mais; ou fumo regularmente); ingestão de bebidas alcóolicas (nunca bebi; bebi, mas não bebo mais; bebo regularmente); $\mathrm{AF}$, estimada por meio do questionário para atividades físicas para crianças 14 e para adolescentes 15 adaptado para a realidade brasileira. ${ }^{16}$ Este questionário incluiu nove questões sobre a prática de esportes, de jogos e realização de AF na escola e durante o lazer, incluindo os finais de semana. Cada questão possui cinco opções de resposta codificadas mediante escala de medida crescente de 1 a 5 pontos ( $1=$ menos ativo; $5=$ mais ativo $)$ possibilitando a obtenção de um escore contínuo a partir da soma das respostas de cada questão. Este escore contínuo foi dividido em tercis $($ tercil $1=$ menos ativos; tercil $3=$ mais ativos)

O estado nutricional e aptidão física dos estudantes foram avaliados pelo cálculo do índice de massa corporal (IMC) e pela aptidão cardiorrespiratória (ApC), respectivamente. As medidas antropométricas (massa corporal e estatura) e o teste de aptidão física foram realizados seguindo protocolos padronizados.17 O IMC foi calculado utilizando equação massa corporal $(\mathrm{Kg}) /$ estatura $(\mathrm{m})^{2}$. Para a medida da ApC foi utilizado o teste de corrida/caminhada de nove minutos. Para classificação do IMC ${ }^{18}$ (peso normal, sobrepeso ou obesidade) e da $\mathrm{ApC}^{17}$ (recomendada e baixa) foram utilizados pontos de corte específicos por sexo e idade.

Para a análise dos dados foram realizadas análises descritivas e análises de associação brutas e ajustadas. Inicialmente foi realizada a descrição da distribuição de frequência absoluta e relativa (proporções) das variáveis estudadas. Em seguida, analisou-se a associação bruta das variáveis independentes com a PAS e PAD elevadas, tendo as diferenças estatísticas sido avaliadas pelos testes do quiquadrado e a magnitude dos efeitos estimada pelo cálculo da odds ratio (OR) bruta e seus respectivos intervalos de confiança de $95 \%$ (IC95\%).

A análise ajustada foi realizada utilizando-se a regressão logística considerando um modelo teórico hierárquico com quatro níveis de determinação para os desfechos. O primeiro nível foi constituído pelas variáveis sociodemográficas (nível distal); o segundo, pelas variáveis psicossociais (nível intermediário/distal); o terceiro pelas variáveis comportamentais (nível intermediário/proximal); e o último nível pelos indicadores de estado nutricional e de aptidão física (nível proximal). Esta forma de análise permite verificar se as associações entre as variáveis independentes e os desfechos são influenciadas por fatores de confusão e de mediação. Variáveis de um nível hierárquico com efeito nas associações de outras variáveis do mesmo nível com o desfecho são consideradas fatores de confusão. ${ }^{19}$ Os fatores de mediação são variáveis que exercem efeito nas associações de variáveis de níveis hierarquicamente inferiores com o desfecho. 19

As variáveis que apresentaram valores de significância inferior a $20 \%(p<0,20)$ nas análises brutas foram incluídas nas análises ajustadas. Nesta análise as associações das variáveis do primeiro nível hierárquico (distal) com os desfechos foram ajustadas apenas para as demais variáveis do mesmo nível. As associações das variáveis dos níveis hierárquicos mais proximais aos desfechos foram 
ajustadas para as variáveis do mesmo nível e para todas as variáveis dos níveis hierárquicos superiores, independentemente dos seus valores de significância. As OR ajustadas e seus respectivos IC 95\% foram calculados para estimar o tamanho do efeito das associações. Permaneceram no modelo hierárquico final as variáveis independentes que após os ajustes se associaram aos desfechos (PAS e PAD elevadas) a um nível de significância de $p<0,05$.

Este estudo foi conduzido respeitando a Resolução 196/96 do Conselho Nacional de Saúde do Ministério da Saúde. O projeto foi aprovado pelo Comitê de Ética em Pesquisa da Universidade Federal do Pampa (Unipampa) (protocolo 042/2010).

\section{Resultados}

De um total de 1554 adolescentes selecionados para o estudo, 41 foram excluídos porque não apresentaram o termo de consentimento livre e esclarecido ou por não estarem presentes no momento da coleta dos dados e 50 porque se recusaram a participar do estudo. Entre os 1463 adolescentes avaliados, oito foram excluídos das análises por estarem fora da faixa etária de interesse. Assim, um total de 1455 escolares, do qual 50,9\% (741) eram meninas, participou do estudo.

As prevalências de PAS e PAD elevadas foram de 16,4\% (IC95\%: 14,4-18,4) e 18,5\% (IC95\%: 16,4-20,6), respectivamente. A Tabela 1 apresenta a distribuição dos resultados da associação bruta das PAS e PAD com as variáveis sociodemográficas, psicossociais, comportamentais e relativas ao estado nutricional e de aptidão física. Adolescentes do sexo feminino $(p=0,008)$, provenientes de famílias de maior nível socioeconômico $(p=0,001)$, residentes em zona rural $(p=0,012)$, que não gostam de praticar atividade física $(p=0,050)$, que se percebem menos em forma e menos ativos que seus pares $(p<0,001)$, que não praticam AF com orientação além da EF escolar $(p=0,011)$, os menos ativos $(p=0,004)$, com sobrepeso ou obesidade $(p<0,001)$ e aqueles com baixa ApC $(p<0,001)$, tiveram mais chance de apresentar PAS elevada. Na mesma análise foi identificado que adolescentes provenientes de famílias de maior nível socioeconômico $(p=0,017)$, que não gostam de praticar AF $(p=0,048)$, que se percebem menos em forma que seus pares $(p<0,001)$ e com sobrepeso ou obesidade $(p<0,001)$, apresentaram chance significativamente superior de apresentar PAD elevada (Tabela 1).

As variáveis "idade" e "gostar e praticar aulas de
EF escolar" se associaram com PAS elevada na análise bruta, a um nível de significância de $p<0,20$ e foram incluídas no modelo de regressão múltipla. Os mesmo ocorreu para as variáveis zona de residência, percepção sobre o nível de $\mathrm{AF}, \mathrm{AF}$ com orientação além da EF escolar, nível de AF e ApC na análise bruta da associação das variáveis independentes com PAD elevada (Tabela 1).

Os resultados da análise de regressão logística após os ajustes realizados de acordo com o modelo teórico hierárquico são apresentados na Tabela 2 . Permaneceram associados à PAS elevada no modelo final $(p<0,05)$ o sexo feminino $\left(\mathrm{OR}_{\text {ajustado }}=1,39\right.$; IC95\%: 1,02-1,90) e ter maior nível socioeconômico $\left(\mathrm{OR}_{\text {ajustado }}=2,39\right.$; IC95\%: 1,31-4,36) entre as variáveis do primeiro nível hierárquico; se perceber menos em forma que seus pares $\left(\mathrm{OR}_{\text {ajustado }}=2,27\right.$; IC95\%: 1,22-4,23) do segundo nível hierárquico; e ter sobrepeso $\left(\mathrm{OR}_{\text {ajustado }}=5,59\right.$; IC95\%: 3,48-8,98) ou obesidade $\left(\mathrm{OR}_{\text {ajustado }}=9,29\right.$; IC95\%:4,89-17,68) do último nível hierárquico. Para a PAD elevada, permaneceram no modelo final $(p<0,05)$ as variáveis "percepção sobre a forma corporal (menos em forma e completamente fora de forma)" $\left(\mathrm{OR}_{\text {ajustado }}=2,99\right.$; IC95\%: 1,34-6,70) do segundo nível hierárquico, e o IMC (sobrepeso - OR: 2,73; IC95\%: 1,76-4,24 e obesidade - OR: 4,70; IC95\%: 2,61-8,45) do quarto nível hierárquico.

\section{Discussão}

As prevalências de PAS e PAD elevadas encontradas entre os adolescentes de Uruguaiana/RS são altas. Porém, valores similares foram relatados por outros estudos realizados no Brasil.8-10 A análise de regressão logística seguindo o modelo teórico hierárquico mostrou que os adolescentes com sobrepeso e obesidade e os que se perceberam menos em forma em relação a seus pares tiveram mais chance de apresentar PAS e PAD elevadas. Do mesmo modo, a chance de apresentar níveis elevados de PAS foi maior entre os adolescentes do sexo feminino e os procedentes de famílias com maior nível socioeconômico.

Estudos prévios realizados no Brasil relataram prevalências inferiores 11,20 e superiores 21 às encontradas no presente estudo. Alguns fatores podem contribuir, ao menos em parte, para estas variações. Embora o método de obtenção tenha sido o mesmo em todos os estudos (método auscultatório), a quantidade de aferições da medida de PA variou entre os estudos, $8-11,20,21$ assim como a metodologia de obtenção da medida da PA. Três estudos $9,11,21$ adotaram o valor médio entre as aferições, um 
Tabela 1

Odds ratio bruta e Intervalo de Confiança de $95 \%$ da associação entre pressão arterial sistólica e diastólica com variáveis sociodemográficas, psicossociais, comportamentais, estado nutricional e de aptidão física em adolescentes de 10 a 17 anos. Uruguaiana, Rio Grande do Sul, 2011.

\begin{tabular}{|c|c|c|c|c|c|c|c|}
\hline \multirow[t]{2}{*}{ Variáveis } & \multirow[t]{2}{*}{$\mathbf{N}$} & \multicolumn{3}{|c|}{ Pressão arterial sistólica elevada } & \multicolumn{3}{|c|}{ Pressão arterial diastólica elevada } \\
\hline & & odds ratio & IC95\% & $p$ & odds ratio & IC95\% & $p$ \\
\hline \multicolumn{8}{|l|}{ Sociodemográficas } \\
\hline Sexo* & & & & 0,008 & & & 0,653 \\
\hline Masculino & 714 & 1 & - & & 1 & - & \\
\hline Feminino & 741 & 1,49 & $1,11-1,99$ & & 1,07 & $0,81-1,41$ & \\
\hline Idade (anos) ${ }^{\dagger}$ & & & & 0,057 & & & 0,373 \\
\hline $10-12$ & 493 & 1,46 & $0,95-2,34$ & & 1,28 & $0,85-1,94$ & \\
\hline $13-15$ & 817 & 1,15 & $0,75-1,76$ & & 1,37 & $0,92-2,04$ & \\
\hline $16-17$ & 198 & 1 & - & & 1 & - & \\
\hline Nível socioeconômico' ${ }^{\dagger}$ & & & & 0,001 & & & 0,017 \\
\hline Classe B & 263 & 2,81 & $1,57-5,03$ & & 1,74 & $1,05-2,88$ & \\
\hline Classe $\mathrm{C}$ & 817 & 1,90 & $1,11-3,24$ & & 1,15 & $0,73-1,80$ & \\
\hline Classe D/E & 198 & 1 & - & & 1 & - & \\
\hline Zona de Residência* & & & & 0,012 & & & 0,169 \\
\hline Urbana & 1296 & 2,21 & $1,17-4,18$ & & 1,44 & $0,86-2,41$ & \\
\hline Rural & 131 & 1 & - & & 1 & - & \\
\hline Tipo de residência* & & & & 0,596 & & & 0,559 \\
\hline Casa & 1345 & 1,19 & $0,62-2,30$ & & 1,21 & $0,64-2,27$ & \\
\hline Apartamento & 84 & 1 & - & & 1 & - & \\
\hline \multicolumn{8}{|l|}{ Psicossociais } \\
\hline \multicolumn{8}{|l|}{ Gostar das aulas de Educação } \\
\hline Física* & & & & 0,073 & & & 0,507 \\
\hline Sim & 1009 & 1 & - & & 1 & - & \\
\hline Não & 265 & 1,39 & $10,97-12,01$ & & 1,13 & $0,79-1,61$ & \\
\hline \multicolumn{8}{|l|}{ Gostar de praticar atividade } \\
\hline física* & & & & 0,050 & & & 0,048 \\
\hline Sim & 1251 & 1 & - & & 1 & - & \\
\hline Não & 178 & 1,50 & $1,00-2,28$ & & 1,49 & $1,00-2,20$ & \\
\hline \multicolumn{8}{|l|}{ Percepção sobre a forma } \\
\hline corporal ${ }^{\dagger}$ & & & & $<0,001$ & & & $<0,001$ \\
\hline Muito mais em forma & 175 & 1 & - & & 1 & - & \\
\hline Mais em forma & 317 & 0,85 & $0,48-1,53$ & & 1,02 & $0,59-1,74$ & \\
\hline Igualmente em forma & 559 & 1,20 & $0,71-2,01$ & & 1,15 & $0,70-1,88$ & \\
\hline Menos em forma & 286 & 2,37 & $1,39-4,04$ & & 2,15 & $1,29-3,58$ & \\
\hline Completamente fora de forma & la 95 & 2,05 & $1,03-4,07$ & & 1,93 & $0,99-3,75$ & \\
\hline \multicolumn{8}{|l|}{ Percepção sobre o nível de } \\
\hline atividade física $^{\dagger}$ & & & & 0,051 & & & 0,100 \\
\hline \multicolumn{7}{|l|}{ Muito menos ativo que os } & \\
\hline Menos ativo que os outros & 286 & 1,83 & $1,01-3,29$ & & 1,41 & $0,83-2,42$ & \\
\hline Igualmente ativo aos outros & 504 & 1,13 & $0,64-2,00$ & & 0,97 & $0,58-1,61$ & \\
\hline Mais ativo que os outros & 301 & 1,22 & $0,67,2,24$ & & 0,88 & $0,50-1,52$ & \\
\hline \multicolumn{8}{|l|}{ Muito mais ativo que os } \\
\hline outros & 139 & 1 & - & & 1 & - & continua \\
\hline
\end{tabular}

$\mathrm{n}=$ número amostral; $\%$ proporção da amostra; $p=$ nível de significância; IC95\% = intervalo de confiança de $95 \%$; *Qui-quadrado para heterogeneidade; ${ }^{\dagger}$ Qui-quadrado para tendência. 
Odds ratio bruta e intervalo de confiança de $95 \%$ da associação entre pressão arterial sistólica e diastólica com variáveis sociodemográficas, psicossociais, comportamentais, estado nutricional e de aptidão física em adolescentes de 10 a 17 anos. Uruguaiana, Rio Grande do Sul, 2011.

\begin{tabular}{|c|c|c|c|c|c|c|c|}
\hline \multirow[t]{2}{*}{ Variáveis } & \multirow[t]{2}{*}{ N } & \multicolumn{3}{|c|}{ Pressão arterial sistólica elevada } & \multicolumn{3}{|c|}{ Pressão arterial diastólica elevada } \\
\hline & & odds ratio & $\mathrm{IC95 \%}$ & $p$ & odds ratio & $\mathrm{IC95 \%}$ & $p$ \\
\hline \multicolumn{8}{|l|}{ Comportamentais } \\
\hline Deslocamento até a escola* & & & & 0,980 & & & 0,417 \\
\hline Ativo & 1082 & 1 & - & & 1 & - & \\
\hline Passivo & 348 & 0,99 & $0,71-1,40$ & & 0,87 & $0,63-1,21$ & \\
\hline \multicolumn{8}{|l|}{ Prática de educação física } \\
\hline escolar* & & & & 0,142 & & & 0,676 \\
\hline Sim & 1191 & 1 & - & & 1 & - & \\
\hline Não & 216 & 1,33 & $0,91-1,90$ & & 1,09 & $0,74-1,60$ & \\
\hline \multicolumn{8}{|l|}{ Atividade física orientada além } \\
\hline da educação física escolar* & & & & 0,011 & & & 0,051 \\
\hline$\leq 1 \mathrm{x} /$ semana & 1059 & 1,66 & $1,12-2,48$ & & 1,44 & $0,99-2,08$ & \\
\hline$\geq 2 \mathrm{x} /$ semana & 305 & 1 & - & & 1 & - & \\
\hline Tempo de tela* & & & & 0,761 & & & 0,348 \\
\hline$\leq 3$ horas & 521 & 1 & - & & 1 & - & \\
\hline$>3$ horas & 934 & 0,95 & $0,71-1,29$ & & 1,15 & $0,86-1,34$ & \\
\hline Atividade física ${ }^{\dagger}$ & & & & 0,004 & & & 0,077 \\
\hline $1^{\circ}$ Tercil & 458 & 1,68 & $1,17-2,41$ & & 1,37 & $0,97-1,94$ & \\
\hline $2^{\circ}$ Tercil & 453 & 1,14 & $0,78-1,67$ & & 1,23 & $0,86-1,74$ & \\
\hline $3^{\circ}$ Tercil & 453 & 1 & - & & 1 & - & \\
\hline Uso de tabaco' ${ }^{\dagger}$ & & & & 0,657 & & & 0,967 \\
\hline Nunca fumou & 1283 & 1 & - & & 1 & - & \\
\hline Fumou, mas não fuma mais & 149 & 0,89 & $0,54-1,17$ & & 1,01 & $0,64-1,60$ & \\
\hline Consumo de bebida alcóolica ${ }^{\dagger}$ & & & & 0,688 & & & 0,221 \\
\hline Nunca bebeu & 803 & 1 & - & & 1 & - & \\
\hline Bebeu, mas não bebe mais & 336 & 1,12 & $0,75-1,66$ & & 0,97 & $0,69-1,36$ & \\
\hline Bebe regularmente & 293 & 1,18 & $0,75-1,86$ & & 0,77 & $0,53-1,13$ & \\
\hline \multicolumn{8}{|l|}{ Estado nutricional e aptidão física } \\
\hline Índice de massa corporal ${ }^{\dagger}$ & & & & $<0,001$ & & & $<0,001$ \\
\hline Peso normal & 990 & 1 & - & & 1 & - & \\
\hline Sobrepeso & 273 & 4,11 & $2,92-5,78$ & & 2,54 & $1,83-3,53$ & \\
\hline Obesidade & 109 & 9,30 & $6,00-14,39$ & & 4,96 & $3,24-7,60$ & \\
\hline Aptidão cardiorrespiratória* & & & & $<0,001$ & & & 0,140 \\
\hline Recomendado & 324 & 1 & - & & 1 & - & \\
\hline Baixa & 926 & 2,17 & $1,44-3,28$ & & 1,29 & $0,92-1,83$ & \\
\hline
\end{tabular}

$\mathrm{n}=$ número amostral; \% proporção da amostra; $p=$ nível de significância; IC95\% = intervalo de confiança de $95 \%$; *Qui-quadrado para heterogeneidade; ${ }^{\dagger}$ Qui-quadrado para tendência. 
Tabela 2

Análise ajustada da associação da pressão arterial sistólica e diastólica elevadas com variáveis sociodemográficos, psicossociais, comportamentais, estado nutricional e de aptidão física em adolescentes de 10 a 17 anos. Uruguaiana, Rio Grande do Sul, 2011.

\begin{tabular}{llccccc}
\hline Variáveis & \multicolumn{3}{c}{ Pressão arterial sistólica elevada } & Pressão arterial diastólica elevada \\
\cline { 2 - 6 } & $\begin{array}{l}\text { odds ratio } \\
\text { ajustado }\end{array}$ & $\mathrm{IC} 95 \%$ & $p$ & $\begin{array}{c}\text { odds ratio } \\
\text { ajustado }\end{array}$ & IC95\% & $p$ \\
\hline
\end{tabular}

Nível distal

Sociodemográficas $(n=1185)$

Sexo

Masculino

Feminino

Idade (anos)

10-12

13-15

16-17

Nível Socioeconômico

Classe B

Classe $C$

Classe D/E

Zona de residência

Urbana

Rural

1
1,39
1,54
1,24
1

$1,02-1,90$

0,039

$0,96-2,44$

0,071

$0,78-1,95 \quad 0,363$

2,39

1,71

$1,31-4,36$

0,004

$0,96-2,95$

0,055

1,60

$0,95-2,69$

0,078

1

1,86

$0,94-3,71$

0,076

1,38

$0,77-2,47$

0,285

Nível intermediário/distal

Psicossociais $(n=1054)$

Gostar das aulas de Educação Física

Sim

Não

09

$0,70-1,69$

0,711

Gostar de praticar atividade física

Sim

Não

\section{1}

Percepção sobre a forma corporal

Muito mais em forma

Mais em forma

Igualmente em forma

Menos em forma

Completamente fora de forma

$0,67-2,00$

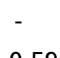

1

0,590

1,02

$0,68-1,52$

0,932

1

$-$

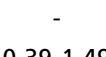

0,428

1

$0,59-1,98$

0,787

1,37

$0,70-2,67$

0,355

1,09

$1,22-4,23$

0,010

1,58

$0,84-2,95$

0,153

$0,97-4,77$

0,059

$1,39-5,08$

0,003

Percepção sobre o nível de

atividade física

Muito menos ativo que os outros 0,93

0,42-2,06

0,861

0,95

0,46-1,96

0,894

$0,78-3,18$

0,206

1,32

$0,69-2,52$

0,402

Igualmente ativo aos outros $\quad 1,04$

$0,53-2,05$

0,907

$0,53-1,84$

0,973

Mais ativo que os outros

1,27

$0,63-2,56$

0,508

0,96

$0,50-1,85$

0,905

Muito mais ativo que os outros

$-$

p= nível de significância; IC95\% = intervalo de confiança de $95 \%$; Amostra no bloco de análise sociodemográfico=1185: Amostra no bloco de análise psicossocial=1054; Amostra no bloco de análise comportamental=957; Amostra no bloco de análise do esta nutricional e de aptidão física=869. 
Análise ajustada da associação da pressão arterial sistólica e diastólica elevadas com variáveis sociodemográficos, psicossociais, comportamentais, estado nutricional e de aptidão física em adolescentes de 10 a 17 anos. Uruguaiana, Rio Grande do Sul, 2011.

\begin{tabular}{|c|c|c|c|c|c|c|}
\hline \multirow[t]{2}{*}{ Variáveis } & \multicolumn{3}{|c|}{ Pressão arterial sistólica elevada } & \multicolumn{3}{|c|}{ Pressão arterial diastólica elevada } \\
\hline & $\begin{array}{l}\text { odds ratio } \\
\text { ajustado }\end{array}$ & IC95\% & $p$ & $\begin{array}{l}\text { odds ratio } \\
\text { ajustado }\end{array}$ & IC95\% & $p$ \\
\hline \multicolumn{7}{|c|}{ Nível intermediário/proximal } \\
\hline \multicolumn{7}{|c|}{ Comportamentais $(n=957)$} \\
\hline \multicolumn{7}{|c|}{ Prática de educação física escolar } \\
\hline Sim & 1 & - & - & - & - & - \\
\hline Não & 1,19 & $0,57-2,49$ & 0,636 & - & - & - \\
\hline \multicolumn{7}{|c|}{$\begin{array}{l}\text { Atividade física orientada além } \\
\text { da educação física escolar }\end{array}$} \\
\hline$\leq 1 \mathrm{x} / \mathrm{semana}$ & 1,41 & $0,87-2,29$ & 0,163 & 1,36 & $0,87-2,13$ & 0,182 \\
\hline$\geq 2 \mathrm{x} /$ semana & 1 & - & - & 1 & - & - \\
\hline \multicolumn{7}{|l|}{ Atividade física } \\
\hline $1^{\circ}$ Tercil & 1,32 & $0,78-2,22$ & 0,297 & 1,21 & $0,74-1,96$ & 0,444 \\
\hline $2^{\circ}$ Tercil & 0,83 & $0,52-1,32$ & 0,439 & 1,06 & $0,69-1,62$ & 0,798 \\
\hline $3^{\circ}$ Tercil & 1 & - & - & 1 & - & - \\
\hline \multicolumn{7}{|l|}{ Nível proximal } \\
\hline \multicolumn{7}{|c|}{ Estado nutricional e de aptidão física $(n=869)$} \\
\hline \multicolumn{7}{|c|}{ Índice de massa corporal } \\
\hline Peso normal & 1 & - & - & 1 & - & - \\
\hline Sobrepeso & 5,59 & $3,48-8,98$ & $<0,001$ & 2,73 & $1,76-4,24$ & $<0,001$ \\
\hline Obesidade & 9,29 & $4,89-17,68$ & $<0,001$ & 4,70 & $2,61-8,45$ & $<0,001$ \\
\hline \multicolumn{7}{|c|}{ Aptidão cardiorrespiratória } \\
\hline Recomendado & 1 & - & - & 1 & - & - \\
\hline Baixa & 1,03 & $0,61-1,75$ & 0,898 & 1,17 & $0,73-1,86$ & 0,516 \\
\hline
\end{tabular}

p= nível de significância; IC95\%= intervalo de confiança de 95\%; Amostra no bloco de análise sociodemográfico=1185; Amostra no bloco de análise psicossocial=1054; Amostra no bloco de análise comportamental=957; Amostra no bloco de análise do esta nutricional e de aptidão física=869.

estudo $^{8}$ utilizou o valor da última (terceira) aferição, e um estudo 20 utilizou o valor mais alto entre as aferições (duas). Ainda, apesar de todos os estudos terem utilizado a mesma proposta 12 de classificação da PA, três deles $8,9,21$ utilizaram os valores acima do percentil 90 como ponto de corte para considerá-la como elevada, enquanto os outros três $10,11,20$ utilizaram os valores acima do percentil 95. Dois estudos ${ }^{11,20}$ que utilizaram como critério o percentil 95 encontraram as menores prevalências de PA elevada, indicando que este resultado pode estar relacionado ao ponto de corte utilizado. No entanto, Gomes e Alves 10 encontraram prevalência similar à do presente estudo mesmo utilizando o percentil 95 como ponto de corte. Todavia, estes autores realizaram apenas uma aferição da medida da PA. Como esta tende a diminuir em aferições consecutivas, 8,20 isto pode auxiliar na compreensão da prevalência encontrada mesmo com o ponto de corte utilizado.

Outra característica que deve ser levada em consideração para a interpretação das prevalências encontradas de PA elevada é o fato das medidas terem sido realizadas em um único dia em todos os estudos. A mensuração em dias diferentes faz com que as prevalências de PA elevada diminuam. 12,22 Esta redução parece estar relacionada a um menor estresse emocional por parte dos adolescentes ao serem avaliados em diferentes oportunidades, que, por estarem mais familiarizados com a medida, 
podem apresentar valores de PA em repouso mais próximos dos reais. 12 Pelo exposto acima, o fato de realizar a medida da PA em um único dia é uma limitação do presente estudo. Contudo, deve-se considerar que em levantamentos populacionais, esta medida, mesmo em apenas um dia, deve ser encorajada, pois serve como triagem de indivíduos com PA elevada.

Diferente da maioria dos estudos citados, $8-11,20$ no presente a PAS e a PAD foram analisadas separadamente, pois evidências indicam que durante a adolescência a hipertensão sistólica isolada é preponderante. ${ }^{23}$ Desta forma, analisar os possíveis fatores associados separadamente para PAS e PAD podem disponibilizar informações adicionais para a compreensão da PA elevada neste período da vida. Neste sentido, alguns estudos 21,22 identificaram que nem todas as variáveis que se relacionam com a PAS se relacionam com a PAD.

Entre os indicadores sociodemográficos analisados, ser do sexo feminino e pertencer à família com nível socioeconômico mais alto se associaram à PAS elevada após os ajustes conforme o modelo teórico hierárquico adotado. Com relação ao sexo, resultados de estudos prévios são controversos. 8 11,21,22 Uma recente revisão envolvendo 55 estudos internacionais também encontrou resultados conflitantes relativos à associação entre o sexo e a PA elevada. 3 Contudo, quando os resultados destes estudos foram analisados a partir uma metaregressão a prevalência de PA elevada foi maior entre os meninos. ${ }^{3}$ No entanto, é importante considerar que o nível de AF da população estudada é menor entre as meninas e estas apresentam uma prevalência superior de excesso de peso. 24 Estas características podem auxiliar na interpretação das razões para o resultado encontrado no presente estudo, pois o sobrepeso e a obesidade estão diretamente associados à PA elevada em adolescentes e a AF parece mediar estas associações. ${ }^{25}$

No que se refere à associação entre o nível socioeconômico e a PA, os resultados de outras investigações ${ }^{8,22}$ vão ao encontro dos achados deste estudo. Como a frequência de indivíduos jovens com sobrepeso e obesidade e fisicamente inativos é superior entre aqueles de famílias com maior nível socioeconômico,26,27 e estas duas variáveis estão relacionadas à PA elevada, ${ }^{25}$ tais associações podem ajudar a explicar, ao menos em parte, o resultado encontrado entre o nível socioeconômico e a PA.

Com relação aos indicadores psicossociais, os resultados da análise de regressão conforme o modelo hierárquico indicaram que adolescentes que perceberam sua forma corporal desfavorável comparada a de seus pares tiveram mais chance de apresentar PA elevada. Adolescentes com sobrepeso e obesidade parecem estar cientes desta condição. ${ }^{28}$ Isto pode ser o primeiro passo para a adesão de adolescentes nesta situação nutricional a programas de perda e controle do peso corporal, contribuindo para inúmeros benefícios a saúde, incluindo o controle da PA.

Não encontramos estudos que tenham investigado a relação da PA elevada com fatores psicossociais similares aos analisados no presente estudo. A inserção de variáveis desta natureza em estudos sobre a PA elevada de adolescentes pode auxiliar para a melhor compreensão deste desfecho, pois estes fatores podem interferir em comportamentos potencialmente associados com a PA elevada em adolescentes, como os hábitos alimentares 9 e a $\mathrm{AF}, 10$ e no sobrepeso e obesidade8-11,20,21 e ApC8 que também se associam ao referido desfecho.

Entre os fatores comportamentais estudados, nenhum se mostrou associado à PA elevada após os ajustes realizados a partir da análise hierárquica. No entanto, é importante destacar que nas análises brutas o nível de $\mathrm{AF}$ e a realização de alguma $\mathrm{AF}$ com orientação além da EF escolar se associaram inversamente à PA elevada. Embora a maior parte dos estudos não tenha encontrado associação significativa entre a AF e a PA de adolescentes, ${ }^{9-11,21}$ as possíveis contribuições deste comportamento no controle da PA desta população não devem ser descartadas.

A AF parece exercer um papel mediador na associação entre sobrepeso, obesidade e PA elevada em adolescentes. $25 \mathrm{Um}$ estudo de intervenção indicou que a prática regular de atividades físicas aeróbicas é capaz de reduzir os valores de PAS, PAD e a prevalência de PA elevada, além de reduzir os valores de IMC, gordura abdominal, aumentar o consumo máximo de oxigênio, e reduzir a espessura da íntima média da artéria carótida e a rigidez arterial. ${ }^{29}$ Desta forma, considerando os inúmeros benefícios à saúde, incluindo aqueles relacionados à PA, programas que ofereçam espaços e oportunidades para a prática regular de AF entre os jovens, além de ações de incentivo para esta prática, devem ser organizados.

Embora no presente estudo a $\mathrm{ApC}$ não tenha se associado à PA elevada após os ajustes realizados pela análise hierárquica, resultados de outras investigações mostraram que adolescentes com baixa $\mathrm{ApC}$ tem mais chance de apresentar PA elevada, mesmo após ajuste pelo IMC e outros fatores de confusão incluídos no modelo de regressão múltipla.8,30 Considerando que a melhoria da $\mathrm{ApC}$ é mediada pelo 
prática de $\mathrm{AF}, 29$ conclui-se que o aprimoramento deste componente da aptidão física deve ser estimulado entre os adolescentes. Para isto, similar ao discutido anteriormente, espaços para a prática e programas de AF devem ser disponibilizados para esta população.

No que se refere ao IMC, os resultados da análise hierárquica mostraram que adolescentes obesos tiveram quase dez vezes mais chance de apresentar PAS elevada e quase cinco vezes mais chance de apresentar PAD elevada quando comparados àqueles com peso normal. Esses resultados concordam com o reportado em outros estudos. 8 11,20-22,25,29,20 Tais evidências reforçam que o sobrepeso e a obesidade são fatores independentemente associados à PA elevada em adolescentes. Apesar da concordância dos resultados entre os estudos, os mecanismos envolvidos nesta associação não estão totalmente estabelecidos. Supõe-se que o sobrepeso e a obesidade induzam o aumento da atividade do sistema nervoso simpático, a resistência à insulina e a uma disfunção endotelial,23,25 os quais explicariam, em parte, o evento.

Com base nestes dados, a elaboração de políticas de controle do peso corporal de crianças e adolescentes torna-se urgente. Para tanto, o poder público e diferentes setores da sociedade, especificamente a escola e a família, precisam unir esforços para traçar e operacionalizar estratégias de combate ao sobrepeso e à obesidade. Não obstante as contribuições deste estudo, algumas limitações e suas possíveis repercussões sobre os resultados devem ser discutidas. Além da obtenção da medida de PA em um único dia, já comentada anteriormente, outra limitação se refere ao desenho do estudo, que não permite estabelecer a associação causal dos

\section{Referências}

1. World Health Organization. Global status report on noncommunicable diseases 2014. WHO Library Cataloguing-in-Publication Data; 2014.

2. Daniels SR. Cardiovascular sequelae of childhood hypertension. Am J Hypertens. 2002; 15: 61S-63S.

3. Moraes ACF, Lacerda MB, Moreno LA, Horta BL, Carvalho HB. Prevalence of high blood pressure in 122,053 adolescents: a systematic review and meta-regression. Medicine (Baltimore). 2014; 93 (27): 1-10.

4. Kollias A, Psilopatis I, Karagiaouri E, Glaraki M, Grammatikos E, Grammatikos EE, Garoufi A, Stergiou GS Adiposity, blood pressure, and carotid intima-media thickness in greek adolescents. Obesity (Silver Spring). 2013; 21 (5): 1013-7.

5. Juonala M, Järvisalo MJ, Mäki-Torkko N, Kähönen M, fatores investigados ao evento. Ainda, o uso de autorrelato para a obtenção das informações comportamentais constitui outra limitação, tornando o estudo vulnerável a viés de informação, uma vez que cientes dos danos potenciais à saúde da inatividade física e de intervalos de tempo prolongados em atividades sedentárias, os entrevistados podem ter superdimensionado a intensidade e frequência da AF e informado menor tempo de uso de televisão, videogame e computadores do que habitualmente realizada. Por fim, fatores potencialmente associados à PA elevada em adolescentes, como genética ${ }^{3}$ e hábitos alimentares 3,10 não foram analisados.

Algumas características que fortalecem os achados do presente estudo devem ser destacadas. $\mathrm{O}$ elevado número de fatores analisados e o fato de ter sido inserido dentre estes fatores psicossociais, ainda pouco explorados em estudos de associação com a PA elevada em adolescentes, são forças discutidas anteriormente. Além destas, o número de adolescentes incluídos no estudo e o procedimento de seleção, que envolveu indivíduos da zona rural e de escolas de diferentes áreas da zona urbana, também devem ser destacados. Tais características conferem precisão e representatividade à amostra, aumentando poder das análises e a validade interna do estudo.

Em conclusão, a prevalência de PA elevada é alta na população estudada. Adolescentes do sexo feminino, de famílias de nível socioeconômico mais alto, que se percebem menos em forma que seus pares, e em situação de sobrepeso e obesidade têm mais chance de apresentar PA elevada. Programas de prevenção à PA elevada em adolescentes devem ser elaborados incluindo ações de combate ao excesso de peso corporal e à inatividade física.
Viikari JSA, Raitakari OT. Risk factors identified in childhood and decreased carotid artery elasticity in adulthood. The cardiovascular risk in young finns study. Circulation. 2005; 112: 1489-96.

6. Lai CC, Sun D, Cen R, Wang J, Li S, Fernandez-Alonso C, Chen W, Srinivasan SR, Berenson GS. Impact of long-term burden of excessive adiposity and elevated blood pressure from childhood on adulthood left ventricular remodeling patterns: the Bogalusa Heart Study. J Am Coll Cardiol. 2014; 64 (15): 1580-7.

7. Yoon E, McCool B, Filipp S, Rocchini A, Kershaw D, Clark S. Pediatric Hypertension Specialists' Perspectives About Adolescent Hypertension Management: Implications for Primary Care Providers. Clin Pediatr (Phila). 2015; 54 (6): 551-6. 
8. Costanzi CB, Halpern R, Rech RR, Bergmann ML, Alli LR, de Mattos AP. Associated factors in high blood pressure among schoolchildren in a middle size city, southern Brazil. J Pediatr (Rio J). 2009; 85 (4): 335-40.

9. Pinto SL, Silva RCR, Priore SE, Assis AMO, Pinto EJ. Prevalência de pré-hipertensão e de hipertensão arterial avaliação de fatores associados em crianças e adolescentes de escolas públicas de Salvador, Bahia, Brasil. Cad Saúde Pública. 2011; 27 (6): 1065-76

10. Gomes BMR, Alves JGB. Prevalência de hipertensão arterial e fatores associados em estudantes de Ensino Médio de escolas públicas da Região Metropolitana do Recife, Pernambuco, Brasil, 2006. Cad Saúde Pública. 2009; 25 (2): $375-81$

11. Silva KS, Farias Júnior JC. Fatores de risco associados à pressão arterial elevada em adolescentes. Rev Bras Med Esporte. 2007; 13 (4): 237-40.

12. NHBPEP (National High Blood Pressure Education Program Working Group on High Blood Pressure in Children and Adolescent). The Fourth report on the diagnosis, evaluation, end treatment of high blood pressure in children and adolescents. Pediatrics. 2004; 114 (2): 555-76.

13. ANEP (Associação Nacional de Empresas de Pesquisa). Critério de classificação econômica Brasil. São Paulo: Associação Nacional de Empresas de Pesquisa (dados com base no levantamento sócio econômico 2009); 2011.

14. Crocker PR, Bailey DA, Faulkner RA, Kowalski KC, Mcgrath R. Measuring general levels of physical activity: Preliminary evidence for the Physical Activity Questionnaire for Older Children. Med Sci Sports Exer. 1997; 29 (10): 1344-9.

15. Kowalski K, Crocker P, Faulkner R. Validation of the physical activity questionnaire for older children. Pediatr Exerc Sci. 1997; 9 (2): 174-86.

16. Silva RR, Malina R. Level of physical activity of in adolescentes of Niterói, Rio de Janeiro, Brazil. Cad Saúde Pública. 2000; 16 (4): 1091-7.

17. Projeto Esporte Brasil. [acesso em 15 mar 2015]. Disponível em: <HTTP://www.proesp.ufrgs.br $>$

18. Cole TJ, Bellizzi MC, Flegal KM, Dietz WH. Establishing a standard definition for child overweight and obesity worldwide: international survey. BMJ. 2000; 320 (7244): 1240-3.

19. Florindo AA, Hallal PC. Epidemiologia da atividade física. 1 ed. São Paulo: Atheneu; 2001.
20. Moura AA, Silva MAM, Ferraz MRMT, Rivera IR Prevalência de pressão arterial elevada em escolares e adolescentes de Maceió. J Pediatr. 2004; 80 (1): 35-40.

21. Araújo TL, Lopes MVO, Cavalcante TF, Guedes NG, Moreira RP, Chaves ES, Silva VM. Análise de indicadores de risco para hipertensão arterial em crianças e adolescentes. Rev Esc Enferm USP. 2008; 42: 120-6.

22. Garcia FD, Terra AF, Queiroz AM, Correia CA, Ramos PS, Ferreira QT, Rocha RL, Oliveira EA. Evaluation of risk factors associated with increased blood pressure in children. J Pediatr. 2004; 80 (1): 29-34.

23. Sorof JM, Poffenbarger T, Franco K, Bernard L, Portman RJ. Isolated systolic hypertension, obesity, and hyperkinetic hemodynamic states in children. J Pediatr. 2002; 140 (6): 660-6.

24. Mello JB, Castagna A, Bergmann MLA, Bergmann GG. Associação entre nível de atividade física e excesso de peso corporal em adolescentes: um estudo transversal de base escolar. Rev Bras Ativ Fis Saúde. 2014; 19 (1): 25-34.

25. Torrance B, McGuire KA, Lewanczuk R, McGavock J. Overweight, physical activity and high blood pressure in children: a review of the literature. Vasc Health Risk Manag. 2007: 3 (1): 139-49.

26. Alves CFA, Silva RDCR, Assis AMO, Oliveira Souza C, Jesus Pinto E, Frainer DES. Fatores associados à inatividade física em adolescentes de 10-14 anos de idade, matriculados na rede pública de ensino do município de Salvador, BA. Rev Bras Epidemiol. 2012; 15 (4): 858-70.

27. Souza MCC, Tibúrcio JD, Bicalho JMF, Rennó HMS, Dutra JS, Campos LG, Silva ES. Fatores associados à obesidade e sobrepeso em escolares. Texto Contexto Enferm. 2014; 23 (3): 712-9.

28. Boa-Sorte N, Neri LA, Leite MEQ, Brito SM, Meirelles AR, Luduvice FBS, Santos JP, Viveiros MR, Ribeiro Jr HC. Maternal perceptions and self-perception of the nutritional status of children and adolescents from private schools. J Pediatr. 2007; 83 (4): 349-56.

29. Farpour-Lambert NJ, Aggoun Y, Marchand LM, Martin XE, Herrmann FR, Beghetti M. Physical activity reduces systemic blood pressure and improves early markers of atherosclerosis in pre-pubertal obese children. J Am Coll Cardiol. 2009; 54 (25): 2396-406.

30. Bergmann GG, Bergmann MLA, Hallal PC. Independent and combined associations of cardiorespiratory fitness and fatness with cardiovascular risk factors in Brazilian youth. J Phys Act Health. 2014; 11 (2): 375-83.

Recebido em 2 de julho de 2015

Versão final apresentada em 18 de setembro de 2015

Aprovado em 1 de outubro de 2015 